\title{
CRISPR/Cas9-mediated mutation of OsSWEET14 in rice cv. Zhonghua11 confers resistance to Xanthomonas oryzae pv. oryzae without yield penalty
}

Xuan Zeng ${ }^{1}$, Yufen Luo ${ }^{1,2}$, Nga Thi Quynh Vu ${ }^{1,2}$, Shujuan Shen ${ }^{1,2}$, Kuaifei Xia ${ }^{1,3}$ and Mingyong Zhang ${ }^{1,3^{*}}$ (D)

\begin{abstract}
Background: Bacterial blight of rice, caused by Xanthomonas oryzae pv. oryzae (Xoo), is a devastating rice disease in Southeast Asia and West Africa. OsSWEET14, encoding a sugar transporter, is known to be a major susceptible gene of bacterial blight targeted by four different transcription activator-like (TAL) effectors from either Asian or African Xoo strains. However, the OSSWEET14 single knockout or promoter mutants in the Kitaake background are moderately resistant or even susceptible to African Xoo strains. Therefore, in this study, we knocked out OsSWEET14 in rice cv. Zhonghua 11 background for disease assessment.

Results: In this study, CRISPR/Cas9 was utilized to disrupt the function of OsSWEET14 by modifying its corresponding coding region in the genome of rice Cv. Zhonghua 11 (CR-S14). In total, we obtained nine different OsSWEET14-mutant alleles. Besides conferring broad-spectrum resistance to Asian Xoo strains, tested mutant alleles also showed strong resistance to African Xoo strain AXO1947. Moreover, the expression of OSSWEET14 was detected in vascular tissues, including the stem, leaf sheath, leaf blade and root. The disruption of OsSWEET14 led to increased plant height without a reduction in yield.

Conclusions: Disruption of OSSWEET14 in the Zhonghua 11 background is able to confer strong resistance to African Xoo strain AXO1947 and Asian Xoo strain PXO86. CR-S14 has normal reproductive growth and enhanced plant height under normal growth conditions. These results imply that CR-S14 may serve as a better tester line than sweet 14 single-knockout mutant in the Kitaake background for the diagnostic kit for rice blight resistance. The genetic background and increased plant height need to be taken into consideration when utilizing OsSWEET14 for resistant rice breeding.
\end{abstract}

Keywords: Bacterial blight, OsSWEET14, Zhonghua 11, AXO1947, Enhanced plant height, No yield penalty

\footnotetext{
* Correspondence: zhangmy@scbg.ac.cn

'Key Laboratory of South China Agricultural Plant Molecular Analysis and Genetic Improvement, Guangdong Provincial Key Laboratory of Applied Botany, South China Botanical Garden, Chinese Academy of Sciences, Guangzhou 510650, China

${ }^{3}$ Center of Economic Botany, Core Botanical Gardens, Chinese Academy of Sciences, Guangzhou 510650, China

Full list of author information is available at the end of the article
}

(C) The Author(s). 2020 Open Access This article is licensed under a Creative Commons Attribution 4.0 International License, which permits use, sharing, adaptation, distribution and reproduction in any medium or format, as long as you give appropriate credit to the original author(s) and the source, provide a link to the Creative Commons licence, and indicate if changes were made. The images or other third party material in this article are included in the article's Creative Commons. licence, unless indicated otherwise in a credit line to the material. If material is not included in the article's Creative Commons licence and your intended use is not permitted by statutory regulation or exceeds the permitted use, you will need to obtain permission directly from the copyright holder. To view a copy of this licence, visit http://creativecommons.org/licenses/by/4.0/ The Creative Commons Public Domain Dedication waiver (http://creativecommons.org/publicdomain/zero/1.0/) applies to the data made available in this article, unless otherwise stated in a credit line to the data. 


\section{Background}

Bacterial blight of rice, caused by Xanthomonas oryzae pv. oryzae $(\mathrm{Xoo})$, is a devastating rice disease in Southeast Asia and West Africa $[1,2]$. The pathogen contains type III effectors that can be injected into rice cells directly via the type III secretion system [3]. Transcription activator-like (TAL) effectors, which are the major virulent effectors in $X o o$, function like eukaryotic transcription factors to induce target gene expression via binding to the effectorbinding elements (EBEs) in the promoter of the target genes $[3,4]$. TAL effectors are composed of an N-terminal type III secretion signal, a C-terminal nuclear-localization signal and activation domain and a central repeat domain. The central repeat region consists of 1.5-33.5 tandem repeats that are typically 33-35 amino acids long, and amino acids at the 12th and 13th positions in each repeat are called repeat variable diresidues (RVDs) [5, 6]. The number and order of the RVDs determine the recognition specificity of TAL effectors [5, 6].

OsSWEETs, which encode a family of sugar transporters, are classified into three clades phylogenetically [7]. OsSWEETs of clade III (OsSWEET11-15) are reported to be able to induce a susceptible response when induced by artificial TAL effectors [7]. Currently, however, only three of them (OSSWEET11, 13 and 14) are known to be induced by Xoo isolated from fields; the corresponding $X o o$ strains that are able to induce the two other OsSWEETs (OSSWEET12 or OsSWEET15) have not yet been identified [8-11]. Moreover, OsSWEET11, 13 and 14 are the major susceptible targets of Xoo [8-10]. OsSWEET11 is targeted by TAL effector PthXo1 and OsSWEET13 by PthXo2 or PthXo2-like TAL effectors, while OsSWEET14 is targeted by four different TAL effectors, i.e., AvrXa7, PthXo3, TalC or Tal5 [7, 8, 10-12]. AvrXa7, PthXo3, PthXo1, PthXo2 and PthXo2-like TAL effectors are present in Asian strains [13]. TalC and Tal5 have only been isolated from African strains, and $\mathrm{TalC}$ exists in all the African Xoo strains sequenced, while Tal 5 is present in half of the strains [13]. Therefore, OsSWEET14 is the target of all sequenced African Xoo strains and most Asian Xoo strains.

Since Xoo activates OSSWEET14 by binding to the specific EBEs in the promoter region, great efforts were invested into generating resistant rice plants by genetic editing of the promoter region of OSSWEET14 or by identifying natural EBE-mutant alleles in germplasm reservoir for resistant rice breeding $[14,15]$. The recessive resistance $(R)$ gene $x a 41(t)$, which is the natural EBEmutational allele of OSSWEET14, has been identified in African rice varieties [16]. $x a 41(t)$ has an $18 \mathrm{bp}$ deletion in the promoter region overlapping with AvrXa7, PthXo3 and Tal5 EBEs, so it confers resistance to Xoo depending on AvrXa7 and PthXo3 for virulence [16]. Since the TalC binding site in the promoter region of $x a 41(t)$ is intact, and all the sequenced African Xoo strains harbor the TalC effector, $x a 41(t)$ is unable to confer resistance to African Xoo strains [13, 16]. Genetically modified rice plants with altered EBEs in the promoter region of OSSWEET14 showed resistance to Xoo depending on the corresponding TAL effector and had normal development $[15,17]$. However, a previous study found that mutations in the TalC EBE in the promoter of OsSWEET14 resulted in a susceptible response to African strain BAI3 that depended on TalC for virulence in the Kitaake background [17]. Recently, researchers found that a mutation in the TalC EBE alone in the Kitaake background still could not confer resistance to African strains; instead a quintuple-mutant promoter lines (rice with mutated PthXo1, PthXo2, TalC, AvrXa7 and Tal5 EBEs) in the Kitaake background were moderately resistant to African strain AXO1947 [13]. Moreover, the sweet14 single-knockout mutant in the Kitaake background was also susceptible to African strain AXO1947, with a median lesion length of approximately $10 \mathrm{~cm}$, whereas the sweet13;sweet14 double-knockout mutant showed complete resistance to African strains [18]. All of these previous studies demonstrated that single knockout of OsSWEET14 in the Kitaake background was unable to confer resistance to African strains. However, OsSWEET11 and OsSWEET13 are not target genes of African Xoo strains. Theoretically, the mutation of those two genes should not contribute to the resistance response of Kitaake to African Xoo strains. The disease response of different mutants cannot be explained with current knowledge.

OsSWEET14, encoding a sugar transporter with seven transmembrane helices, targets the plasma membrane and is mainly responsible for sucrose and glucose transportation [19, 20]. TAL effectors function in diverting the nutritional resources from rice by inducing the expression of OSSWEET14 [19-24]. The SWEET11 and SWEET12 single mutant of Arabidopsis did not show obvious morphological defects [20]. However, the double mutant was smaller and had an impaired ability to export sucrose from the leaves [20]. Although the rice OsSWEET14 T-DNA insertion mutant showed AvrXa7- and PthXo3-specific recessive resistance, the homozygous mutant had dramatic development defects including small seeds and severe growth retardation [9]. The homozygous plants required $\sim 30$ more days to reach the size of 14-day-old normal plants [9].

In our study, we used CRISPR/Cas9 to mutate the coding region of OSSWEET14 in rice cv. Zhonghua 11 $(C R-S 14)$ in order to test whether the disruption of OsSWEET14 in the Zhonghua 11 background will result in broad-spectrum resistance to Xoo strains including those originate from Africa. This will help us to know whether CR-S14 is a better tester line than sweet14 single- 
knockout mutant in the Kitaake background for the diagnostic kit for rice blight resistance and also whether the genetic background needs to be taken into consideration when utilizing OsSWEET14 for resistant rice breeding. Moreover, the assessment of CR-S14 agronomic traits will help us understand the role of OsSWEET14 in development and will provide more information when utilizing the OSSWEET14 knockout mutant for resistant rice breeding.

\section{Results}

Generation of rice lines edited in the OSSWEET14 coding region (CR-S14)

To modify OsSWEET14 in rice cv. Zhonghua 11, a CRISPR/Cas9 construct targeting two targets in the corresponding coding region of the OsSWEET14 genome sequence was built and transformed into Zhonghua 11 background. Target I and Target II were located in the 1st and 3rd exon, respectively (Fig. 1a). Polymerase chain reaction $(\mathrm{PCR})$ and sequencing were used to detect the modifications in the rice transformants. Two rice lines, CR-S14-2 and CR-S14-6, harbored homozygous mutant alleles in the $\mathrm{T}_{0}$ generation (Fig. 1b), and the other four lines contained biallelic mutant alleles in the $\mathrm{T}_{0}$ generation, i.e., $C R-S 14-1, C R-S 14-9, C R-S 14-10$ and CR-S14-29; homozygous mutant alleles were obtained in the $\mathrm{T}_{1}$ or $\mathrm{T}_{2}$ generation. According to the sequencing result, the modification in mutant allele $C R$ S14-1-I was the same as that in CR-S14-6, so a total of nine heritable mutant alleles were obtained (Fig. 1b).
A Target I:

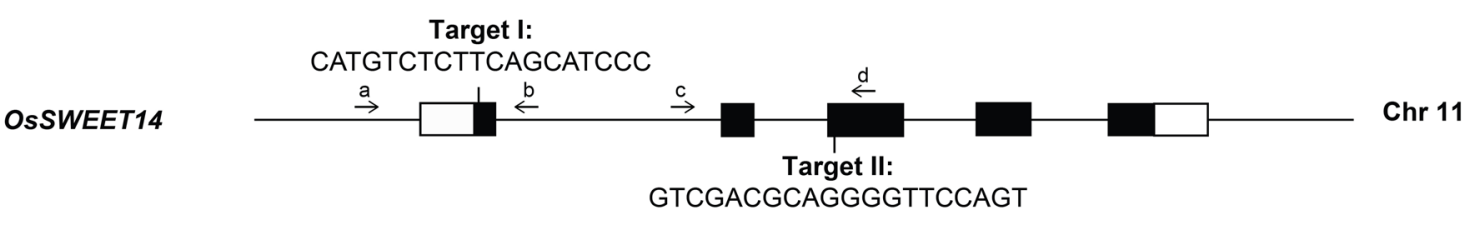
B
OsSWEET14 atggctggCATGTCTCTTCAGCATCCCtgggccttcgectttg .
CR-S14-1-I atggetggCATGTCTCTTCAGCATCCCtgggecttcgcctttg
CR-S14-1-II atggctggCATGTCTCTTCAGCATCCCtgggecttcgcctttg
CR-S14-2 atggctggCATGTCTCT- . . . . - . gggcttcgcctttg
CR-S14-6 atggctggCATGTCTCTTCAGCATCCCtgggccttcgcctttg
CR-S14-9-I atgge
CR-S14-9-II atggctggCATGTCTCTTCAGCC - - CCtgggccttcgcctttg CR-S14-10-I atggctggCATGTCTCTTCAGCATCCCtgggecttcgcctttg CR-S14-10-II atggctggCATGTCTCTTCAGCAT - - - -gggccttcgcctttg
CR-S14-29-I atggetggCATGTCTC - . . . . - . CCCtgggccttcgcctttg
CR-S14-29-II atggctggCАTGTCTC . . . . . . . CCCtgggcettcgcctttg

$$
\begin{aligned}
& \text { agcaaGTCGACGCAGGGGTTCCAGTcgg WT } \\
& \text { Target II } \\
& \text { agcaaGTCGACGCAGGGGTTCCÁATcgg +1 bp } \\
& \text { agcaaGTCGACGCAGGGGTTC - AGTcgg -1 bp } \\
& \text { agcaaGTCGACGCAGGGGTTCCÁATcgg }-11+1 \text { bp } \\
& \text { agcaaGTCGACGCAGGGGTTCCAGTcgg +1 bp } \\
& \begin{array}{ll}
\multicolumn{2}{c}{\text { AA }} \\
\text { agcaaGTCGACGCAGGGGTTCCAGTcgg } & -33+2 \text { bp } \\
\text { agcaaGTCGACGCAGGGGTTCCAGTcgg } & -2+1 \text { bp }
\end{array} \\
& \begin{array}{cc}
\stackrel{\text { AAA }}{\text { agcaaGTCGACGCAGGGGTTCCAGTcgg }}+3 \mathrm{bp} \\
\text { agcaaGTCGACGCAGGGGTTCCAGTcgg } & -4+1 \mathrm{bp}
\end{array} \\
& \text { agcaaGTCGACGCAGGGGTTCCAGTcgg }-8+1 \text { bp } \\
& \text { agcaaGTCGACGCAGGGGTTC - AGTcgg -8-1 bp }
\end{aligned}
$$

Fig. 1 Genetic modification of OsSWEET14 using CRISPR/Cas9. a Two targets were selected in the coding region of OsSWEET14. Target I is located in the 1st exon, while target II is located in the 3rd exon. Two pairs of primers ( $a$ and b, $c$ and d) were used to amplify the two target regions for sequencing. Black boxes and white boxes represent the corresponding CDS and untranslated region of the OsSWEET14 transcript in the genome, respectively. a, Target-TalC-F; b, Target-TalC-R; c, Target-S14E3-F; and d, Target-S14E3-R. b Sequences of OsSWEET14 mutant alleles in the target regions. Mutations in CR-S14-2 and CR-S14-6 were homozygous in the $T_{0}$ generation, while mutations in CR-S14-1, CR-S14-9, CR-S14-10 and CRS14-29 were biallelic in the $T_{0}$ generation. All the biallelic mutations were homozygous in the $T_{1}$ or $T_{2}$ generation. CR-S14-1-I was the same as CR-S14-6. Deletions and insertions are indicated by dashes and red letters, respectively. Target I and Target II are underlined in the wildtype (WT), The number of nucleotides that were deleted or inserted in mutant alleles is indicated on the right side of the mutant allele sequence 
Six of the mutant alleles contained frameshift mutations, i.e., CR-S14-1-II, CR-S14-2, CR-S14-6, CR-S14-9I, CR-S14-9-II and CR-S14-29-I. The three other mutant alleles, CR-S14-10-I, CR-S14-10-II and CR-S14-29II, contained in-frame mutations (Fig. 1b). Mutations were also amplified and confirmed in several mutant transcripts (Additional file 1). Since OsSWEET14 is a sugar transporter containing seven transmembrane helices, the function of the protein depends strongly on the helices. The TMHMM2.0 program was utilized to predict the transmembrane helices of proteins encoded by mutant alleles [25]. All of the frameshift mutant alleles encoded proteins with only one or even no transmembrane helices, while all the in-frame mutant alleles encoded proteins containing five or six transmembrane helices (Additional files 2 and 3). Loss of the transmembrane helices had high possibilities of disrupting the transporter activity. This may indicate that all the OsSWEET14 mutant alleles encode proteins without sugar transportation ability.

\section{CR-S14 conferred strong resistance to African Xoo strain AX01947}

Zhonghua 11 contained the recessive resistance allele of OsSWEET13, which was a deletion in the PthXo2 EBE region leading to the incapability of being recognized by PthXo2 (Additional file 4). PXO86 is a Philippinesoriginated Xoo that depended on AvrXa7 to activate OSSWEET14 expression for virulence, while T7174 (NCBI: txid342109) is a Japanese strain harboring both AvrXa7 and PthXo2 for virulence. Consistent with OsSWEET14 knockout mutant in the Kitaake background reported previously, CR-S14 conferred strong resistance to both PXO86 and T7174 (Additional file 5 and Table 1) [18]. In addition, CR-S14 showed a broad resistance to the tested Asian Xoo strains (Table 1).

To test the disease response of CR-S14 to Xoo strains that depended on TalC for virulence, an Africaoriginated Xoo strain (AXO1947) harboring TalC was inoculated on CR-S14 and Zhonghua 11 by the leaf clipping method. At least three plants of each mutant allele were inoculated with AXO1947. Fourteen days after inoculation, Zhonghua 11 showed a susceptible response to AXO1947, while CR-S14 conferred strong resistance to AXO1947 with an average lesion length less than 2 $\mathrm{cm}$ (Fig. 2). This demonstrated that the disruption of OsSWEET14 in the Zhonghua 11 background conferred strong resistance to AXO1947.

To determine whether the mutant alleles were still inducible, PXO86 and sterile $\mathrm{H}_{2} \mathrm{O}$ were inoculated on $C R$ S14 and Zhonghua 11 with syringes. The expression level of OSSWEET14 and the mutant alleles were measured at 0 and $48 \mathrm{~h}$ after inoculation using qRT-PCR. PXO86 was able to induce the expression of both
Table 1 Disease resistance evaluation of Xoo strains from different regions. At least 10 leaves of three plants were inoculated for each Xoo strain

\begin{tabular}{|c|c|c|c|}
\hline \multirow[t]{2}{*}{ Strain $^{a}$} & \multirow{2}{*}{$\begin{array}{l}\text { Strain } \\
\text { origin }\end{array}$} & \multicolumn{2}{|c|}{ Lesion length and disease response ${ }^{b}$} \\
\hline & & Zhonghua 11 & CR S14-6 \\
\hline GD1358 & China & $8.11 \pm 3.86$ (MS) & $0.26 \pm 0.29(\mathrm{R})$ \\
\hline JS49-6 & China & $11.9 \pm 2.50(\mathrm{~S})$ & $0.87 \pm 0.72(R)$ \\
\hline HB17 & China & $19.5 \pm 8.23(\mathrm{~S})$ & $1.74 \pm 16.1(\mathrm{R})$ \\
\hline HB21 & China & $15.05 \pm 5.85(S)$ & $0.14 \pm 0.06(R)$ \\
\hline HLJ72 & China & $12.91 \pm 4.87(\mathrm{~S})$ & $0.65 \pm 0.72(R)$ \\
\hline NX42 & China & $13.00 \pm 7.24(S)$ & $0.63 \pm 0.88(R)$ \\
\hline HN1-2 & China & $16 \pm 5.62(S)$ & $0.7 \pm 1.12(\mathrm{R})$ \\
\hline LC-4 & China & $17.05 \pm 7.26(S)$ & $1.04 \pm 1.25(\mathrm{R})$ \\
\hline IV-1 & China & $14.64 \pm 5.36(S)$ & $0.43 \pm 0.43(\mathrm{R})$ \\
\hline PXO79 & Philippines & $7.26 \pm 2.86$ (MS) & $0.49 \pm 0.42(R)$ \\
\hline PXO86 & Philippines & $8.17 \pm 3.05$ (MS) & $0.25 \pm 0.31(R)$ \\
\hline PXO71 & Philippines & $8.59 \pm 2.94$ (MS) & $10.27 \pm 2.44(S)$ \\
\hline Aust2031 & Australia & $8.78 \pm 5.53$ (MS) & $0.24 \pm 0.25(R)$ \\
\hline T7174 & Japan & $8.27 \pm 8.44$ (MS) & $0.35 \pm 0.28(R)$ \\
\hline A3857 & India & $10.57 \pm 3.21(S)$ & $10.19 \pm 3.61(\mathrm{~S})$ \\
\hline A3842 & India & $10.11 \pm 2.47(S)$ & $11.66 \pm 2.48(S)$ \\
\hline
\end{tabular}

${ }^{a}$ Xoo strains were cultivated two days on PSA medium and inoculated on sixweek-old rice plants

${ }^{\mathrm{b}}$ Lesions length was scored 14 days after inoculation. $\mathrm{R}$, resistant, lesion length $<3.0 \mathrm{~cm}$; MS, moderately susceptible, $6.0 \mathrm{~cm}<$ lesion lenght $\leq 9.0 \mathrm{~cm} ; \mathrm{S}$, susceptible, lesion length $>9.0 \mathrm{~cm}$

' CR S14-6, one of the homozygous OsSWEET14 knockout mutant in Zhonghua 11 background

mutant alleles in CR-S14 and OsSWEET14 in Zhonghua 11 (Additional file 5). This result demonstrated the mutant alleles were still inducible by AvrXa7, and the resistance response was caused by the disruption of OsSWEET14 transporter activity.

\section{CR-S14 had enhanced plant height and normal reproductive growth}

OsSWEET14 transcripts were detected in various tissues of rice plants using qRT-PCR; the highest levels were measured in the stem, followed by leaf sheaths and blades (Fig. 3a). The expression pattern of OsSWEET14 was further investigated in pOsSWEET14:GUS transgenic rice plants using a $\beta$-glucuronidase (GUS) reporter gene under the control of the OSSWEET14 promoter. Consistent with the qRT-PCR result, strong GUS activity was detected in most of the cell types in the stem and mainly in the veins of leaf sheath and blades (Fig. 3b and d). Consistent with the expression pattern of OsSWEET14 in the RiceXPro database [26], GUS activity was also highly detected in the roots; however, root tips, which do not have vascular bundles, lacked GUS activity (Fig. 3g). Low GUS activity was detected in the spikelet, including the palea, lemma and anther (Fig. 3e and f). 


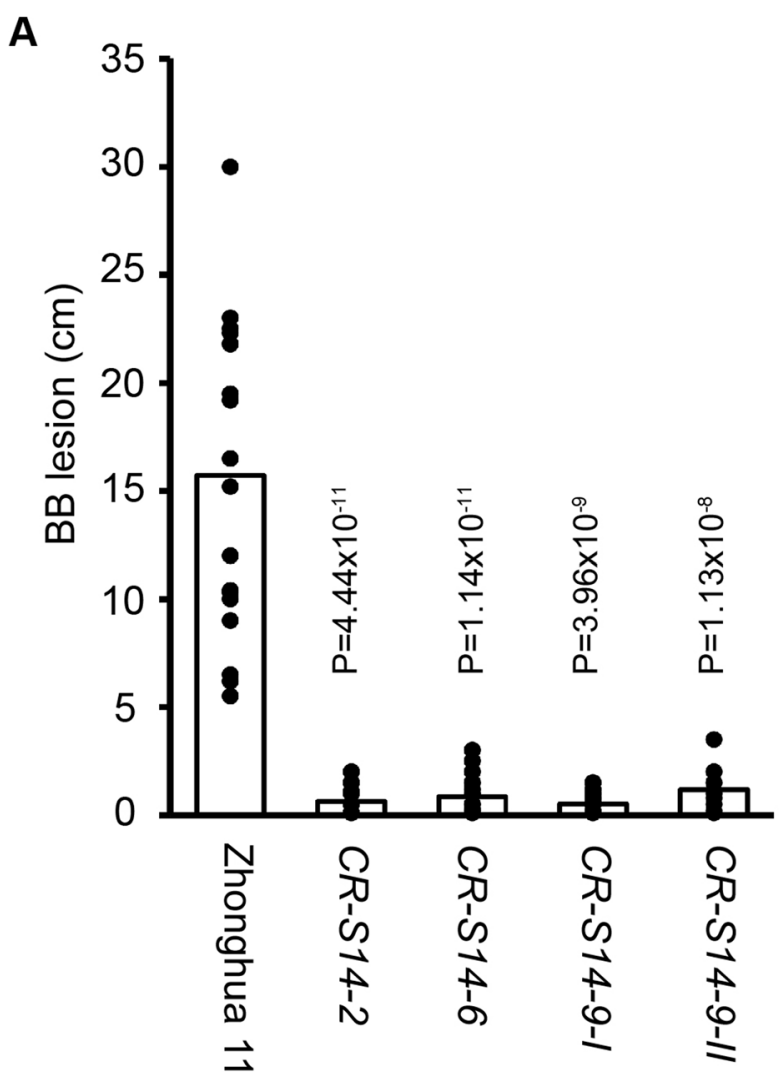

B

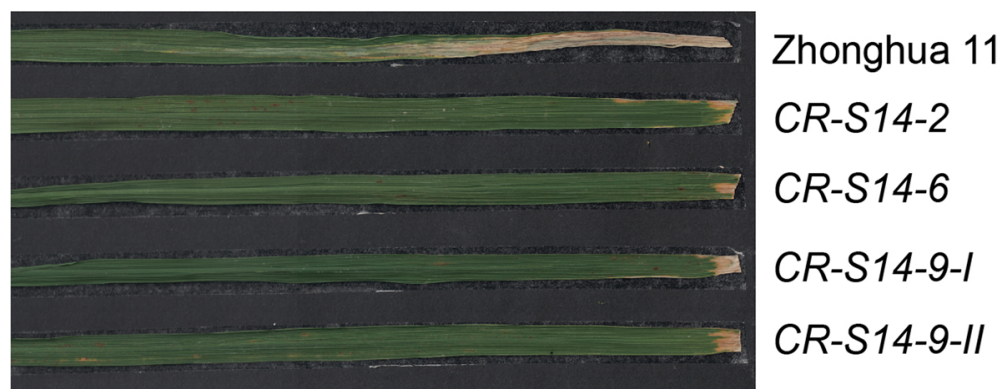

Fig. 2 CR-S14 confers strong resistance to AXO1947. a Lesion lengths of CR-S14 and Zhonghua 11 inoculated with AXO1947 that harbored TalC at 14 days after inoculation $(n>15)$. b Phenotype of CR-S14 and Zhonghua 11 at 14 days after inoculation with AXO1947. CR-S14-2, CR-S14-6, CRS14-9-I and CR-S14-9-II rice plants harboring different homozygous OsSWEET14 mutant alleles in the Zhonghua 11 background. Zhonghua 11, wildtype control. Six-week-old rice plants were inoculated with AXO1947. Lesion lengths on inoculated leaves were scored 14 days after inoculation. Statistical analysis was performed using a two-tailed Student's $t$ test against Zhonghua 11

This indicated that OsSWEET14 is mainly expressed in the vascular tissues of rice plants.

To verify the influence of OSSWEET14 disruption on rice development, CR-S14 and Zhonghua11 were grown in a paddy field in Guangdong Province in China. $C R$ S14 and Zhonghua 11 were germinated and transferred to the paddy field at the same time, and no growth retardation was observed (Fig. 4a). Since OsSWEET14 transcripts accumulated highly in the stem, we tested whether the knockout of OSSWEET14 affected the stem diameter and plant height at the mature stage. Statistical analysis was performed using a two-tailed Student's $t$ test against Zhonghua 11. No significant difference $(P>$ $0.05)$ in stem diameter was detected between CR-S14 and Zhonghua 11 (Fig. 4c and Additional file 6). However, CR-S14-2, CR-S14-9-I and CR-S14-9-II were approximately $7 \mathrm{~cm}$ taller than Zhonghua 11 , which equates to an approximate $8 \%$ increase in plant height (Fig. 4b and Additional file 6). The stem diameter and plant height were recorded for two seasons in 2019 in Guangdong, China. This suggested that the disruption of OSSWEET14 led to an increase in plant height. 

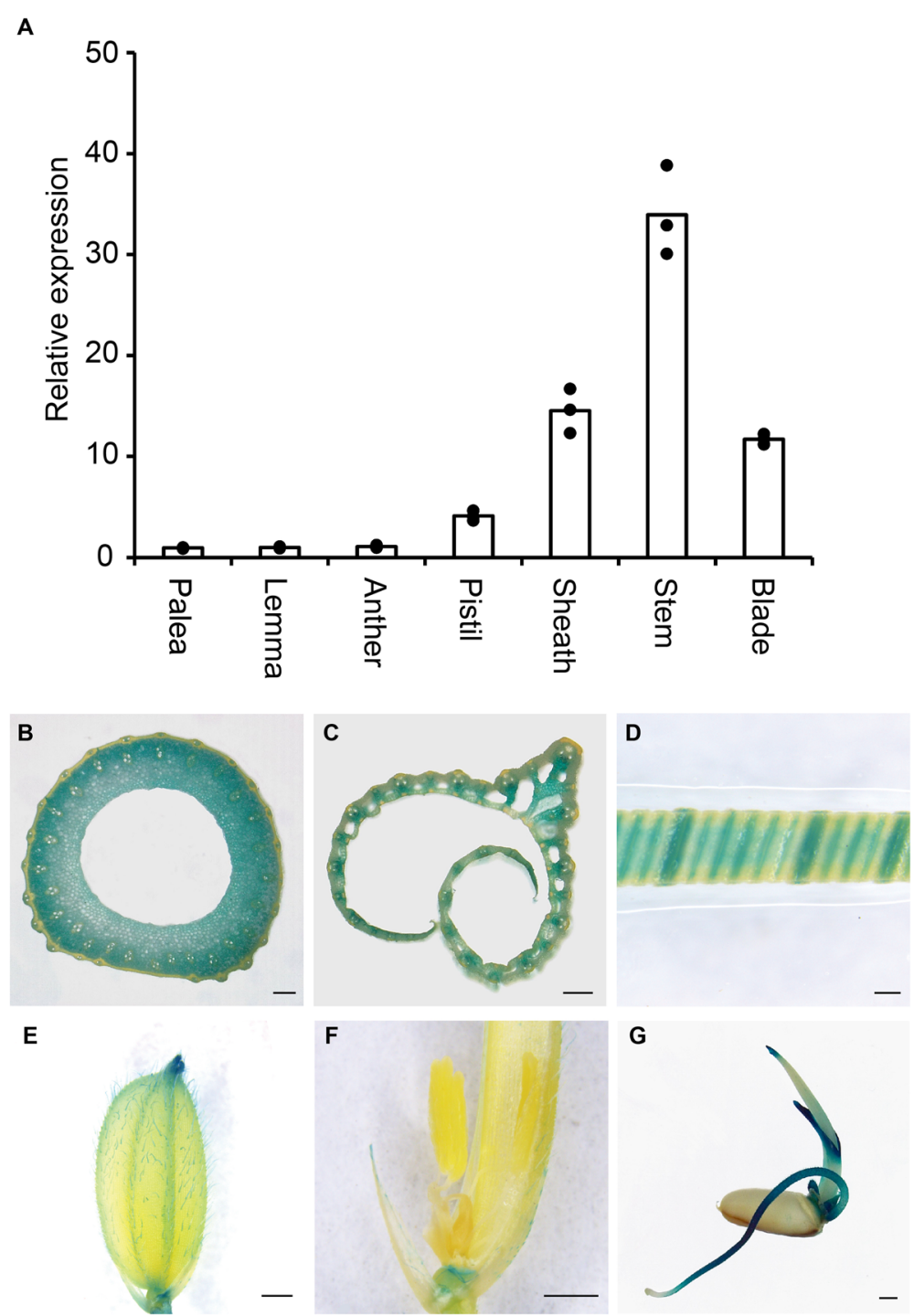

Fig. 3 Tissue-specific expression of OsSWEET14. a Detection of OsSWEET14 transcripts in different rice tissues by qRT-PCR. b-g GUS staining in different tissues of pOsSWEET14:GUS transgenic plants in the Zhonghua 11 background, including the stem (b), leaf sheath (c), leaf blade (d), spikelet (e), anther (f) and seedling (g). Scale bars, $250 \mu \mathrm{M}(\mathbf{b}-\mathbf{d})$ and $1 \mathrm{~mm}(\mathbf{e}-\mathbf{g})$

In addition, we checked whether the disruption of OSSWEET14 affected the reproductive growth of rice. At least 15 plants of each mutant allele and 30 Zhonghua 11 plants were grown in the paddy field in Guangzhou, Guangdong, China. The 1000-grain weight, seed setting rate and yield of the main panicles were assessed. The assessment was executed for two seasons in 2019. The 1000-grain weight of CR-S14-2 and CR-S14-9-II was slightly higher $(P<0.05)$ than that of Zhonghua 11 , while CR-S14-6 and CR-S14-9-I did not differ significantly $(P>0.05)$ from Zhonghua 11 (Fig. 5 and Additional file 6). This suggested that the disruption of OsSWEET14 did not affect the reproductive growth of rice plants under normal growth conditions. All these results indicated that the disruption of OSSWEET14 increased plant height without reducing yield under normal growth conditions.

\section{Discussion}

In natural germplasm reservoirs, recessive resistant alleles that harbor disrupted EBEs in the promoter region are able to confer resistance to Xoo strains depending on the corresponding TAL effectors for virulence. However, TAL effectors are able to adapt to the recessive resistance alleles under the selection pressure from the resistant varieties. Recently, many PthXo2-like, AvrXa7/ PthXo3-like, TalC-like and PthXo1-like TAL effectors have been identified in Xoo strains that are able to activate the expression of OsSWEET13, OsSWEET14 or OsSWEET11 in different rice varieties [12, 13]. For example, 


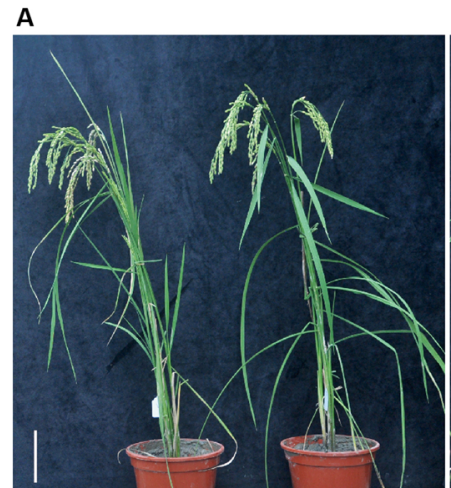

Zhonghua $11 \quad$ CR-S14-2

B

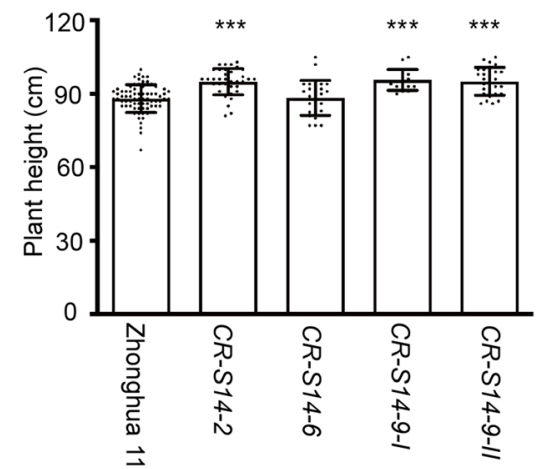

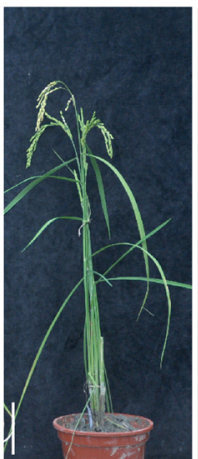

CR-S14-6
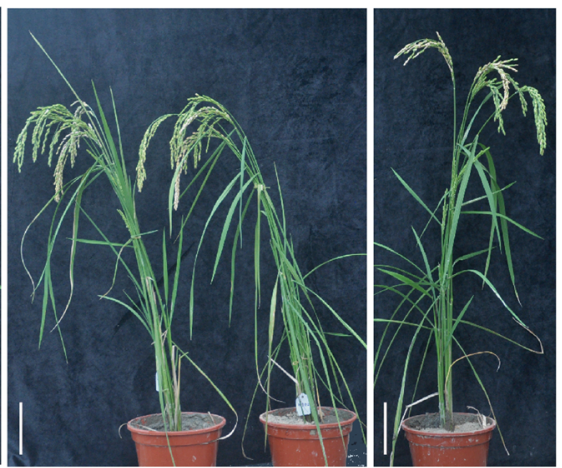

Zhonghua 11 CR-S14-9-I CR-S14-9-II

C

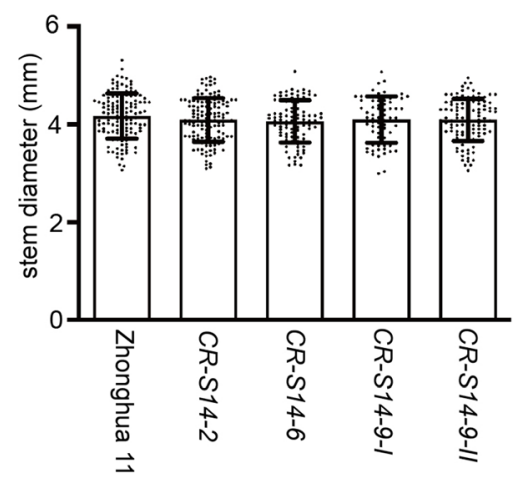

Fig. 4 CR-S14 had enhanced plant height. a CR-S14 did not show obvious morphological defects under normal growth conditions at the mature stage. b, c Performance of CR-S14 plants in terms of height (b) and stem diameter (c) relative to Zhonghua 11 plants at the mature stage. CRS14-2, CR-S14-9-I and CR-S14-9-II were significantly taller than Zhonghua $11(P<0.001)$. The plant height of CR-S14-6 was comparable to that of Zhonghua $11(P>0.05)$. No significant difference was detected in the stem diameter between mutant lines and Zhonghua $11(P>0.05)$. Scale bar, $10 \mathrm{~cm}$. Statistical analysis was performed using a two-tailed Student's $t$ test against Zhonghua 11, and the significance is indicated by asterisks as follows: $0.01<P<0.05\left(^{*}\right), 0.001<P<0.01\left(^{(*)}, P<0.001\left(^{* * *}\right) . n>15\right.$

OSSWEET13 $3_{\text {Kit }}$ in the Kitaake background, which is the natural EBE-mutational allele of OsSWEET13, is noninducible by PthXo2. However, two PthXo2-like TALE effectors (Tal5 $5_{\mathrm{LN} 18}$ and Tal7 $\left.7_{\mathrm{PXO} 1}\right)$ identified recently are able to activate the expression of OsSWEET13 $3_{\text {Kit }}$ to induce susceptible response [12]. These results indicate that recessive resistance can be overcome by the emergence of novel TAL effectors, and the engineering of the promoter region of OSSWEETs genes is not sufficient to confer durable and broad-spectrum resistance to Xoo. As a result, we directly knocked out OsSWEET14 in the Zhonghua 11 background directly (CR-S14) in order to confer resistance to all the Xoo strains that depend on OSSWEET14 induction for virulence since Zhonghua 11 contains the recessive PthXo2 EBE mutant allele (Additional file 4). CR-S14 was able to confer complete resistance to strains depending on both PthXo2 and AvrXa7 for virulence, such as Xoo strain T7174 (Table 1). Furthermore, CR-S14 also conferred complete resistance to African strain AXO1947, which depends on TalC for OsSWEET14 induction. However, the disease response was inconsistent with that in the Kitaake background. OsSWEET14 knockout mutants or OsSWEET14 promoter mutants in the Kitaake background are susceptible to AXO1947 [13, 18]. This inconsistence implies other susceptible targets could be induced in the Kitaake genetic background instead of Zhonghua 11. In 2018, one research paper reported that TAL effector TalB $\mathrm{B}_{\text {MAI1 }}$ from the African Xoo strain MAI1 was able to activate the expression of two susceptible genes (OsTFX1 and OsERF\#123) in the Nipponbare background and induce a susceptible response [27]. This suggested that African Xoo strains might be able to induce other susceptible genes in the Kitaake background besides OsSWEET14 for virulence. The OsSWEET14 single knockout mutant in the Kitaake background was susceptible to AXO1947. This indicated that the genetic background of rice varieties may affect the resistance response of the OsSWEET14 knockout mutant. This hypothesis can be verified by hybridizing CR-S14 with Kitaake and checking the resistance response of $\mathrm{F}_{2}$ generation plants. If novel susceptible targets exist in the Kitaake background, 

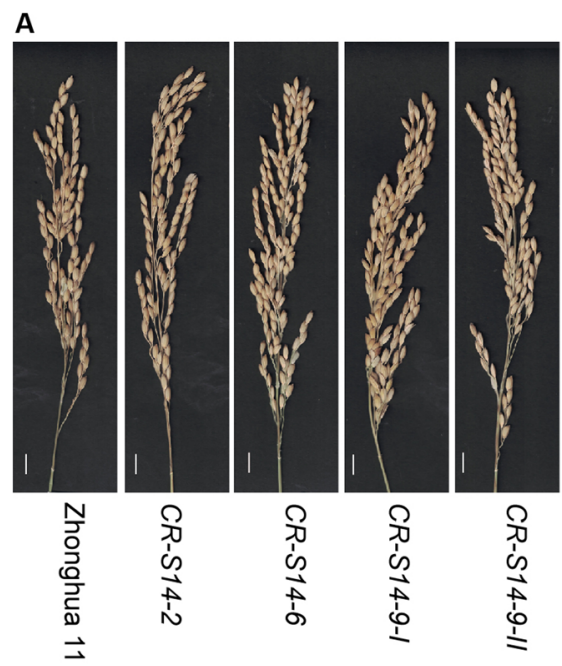

C

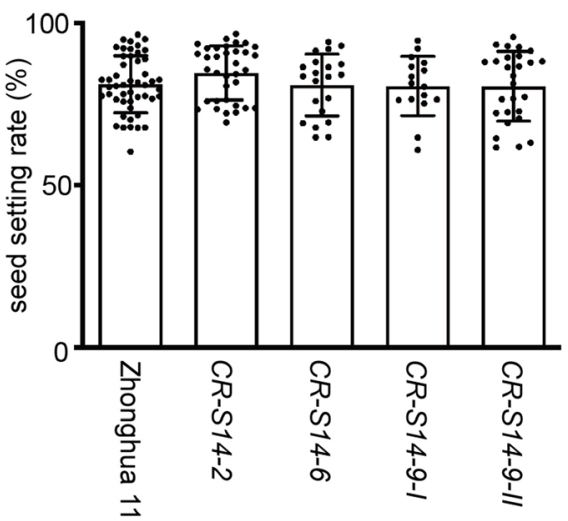

B

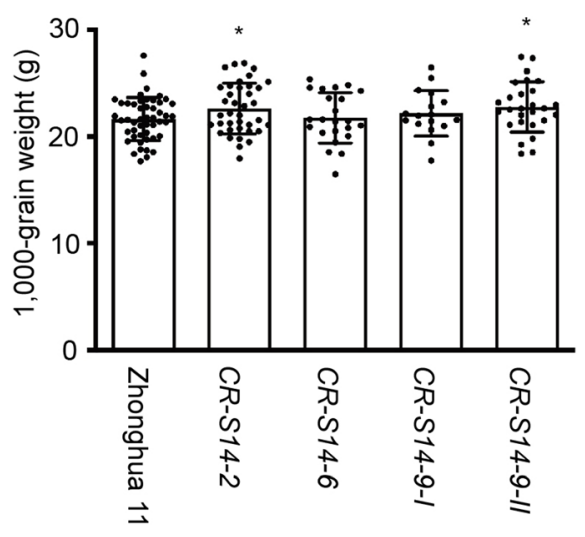

D

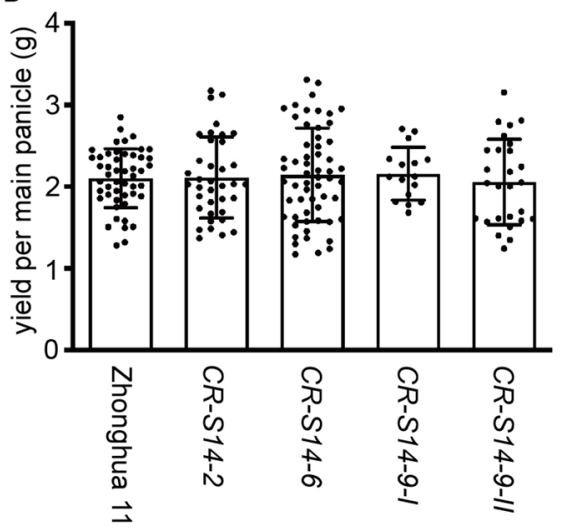

Fig. 5 CR-S14 had normal reproductive growth in the paddy field. a A comparison of the main panicles at the mature stage. No obvious defects were observed under normal growth conditions. b-d 1000-grain weight (b), seed setting rate (c) and yield (d) of the CR-S14 main panicle relative to that of Zhonghua 11 at the mature stage. The 1000-grain weight of CR-S14-2 and CR-S14-9-II was slightly higher than that of Zhonghua 11 $(0.01<P<0.05)$. CR-S14-6 and CR-S14-9-1 had a similar 1000-grain weight to that of Zhonghua 11. No significant difference between CR-S14 and Zhonghua 11 was detected in the seed setting rate and yield. Scale bar, $1 \mathrm{~cm}$. Statistical analysis was performed using a two-tailed Student's $t$ test against Zhonghua 11, and the significance is indicated by asterisks as follows: $0.01<P<0.05\left({ }^{*}\right), 0.001<P<0.01\left(^{* *}\right), P<0.001\left(^{(* *}\right) . n>15$

$\mathrm{F}_{2}$ generation plants harboring the homozygous CR-S14 allele will show disease-response phenotype segregation. Another possibility causing this disease response difference is the off-target effect. Mutagenesis of OsSWEET14 in the Zhonghua 11 background was mediated by CRISPR/Cas9 targeting of two target sites, Target I was in the 1st exon, and Target II was in the 3rd exon (Fig. 1). Mutagenesis of OSSWEET14 in the Kitaake background was mediated by only one target site that is the same site as Target I in Zhonghua 11 (Kittake target: 5'-GCATGTCTCTTCAG CATCCCTGG-3' vs. Target I (CR-S14): 5'-CATGTC TCTTCAGCATCCCTGG-3'). This implies that the offtarget effect of Target II in Zhonghua 11 might lead to a difference in the disease response. This can be verified by generating a new OsSWEET14 knockout mutant in Zhonghua 11 background with CRISPR/Cas9 targeting to Target I or other different target sites.
TalC and Tal5 are two TAL effectors that target different EBEs in the promoter region of OsSWEET14. All the sequenced African Xoo strains habor TalC or both TalC and Tal5, which indicates that all the African Xoo strains are able to activate OSSWEET14 [13]. The OsSWEET14 knockout mutant in the Kitaake background is susceptible to Africa-originated Xoo strains, while CR-S14 is able to confer strong resistance to AXO1947. This implies $C R-$ S14 may serve as a better tester line than sweet14 singleknockout mutant in the Kitaake background for the diagnostic kit for rice blight resistance [18].

CR-S14 plants were taller than Zhonghua 11 and had no reproductive defects under normal growth condition. Since OsSWEET14 had the highest expression level in the stem, the enhanced plant height might be due to the lower efficiency of sugar transportation in the stem, which needs to be studied further. In addition, 
OsSWEET14 may not be responsible for reproductive development since its expression in the anther, palea, lemma and pistil was relatively low. These results imply the enhancement of plant height needs to be taken into consideration if knockout mutants of OsSWEET14 are used to confer resistance to bacterial blight. The sweet14 single-knockout mutant in the Kitaake background also showed normal growth as reported [18]. However, the homozygous OsSWEET14 T-DNA insertion mutant showed dramatic developmental defects [9]. This might be due to multiple T-DNA insertions in the rice genome, which were not discussed in paper [9].

Through comparison with the 3,010 rice genomes from 3,000 Rice Genome Project [28], we found most SNPs or indels in the OSSWEET14 genome region were in untranslated regions or introns. Two and four SNPs were identified in the 3rd and 5th exons, respectively, and 23 different indels were found in the 5th exon. However, all the indels are in the $\mathrm{C}$ terminal of OsSWEET14 and do not affect the transmembrane helices. Therefore, whether those indels abrogate the function of OsSWEET14 needs to be tested further. In addition, the natural OSSWEET14 non-functional alleles need to be discovered.

\section{Conclusions}

This study demonstrated that the disruption of OsSWEET14 in the Zhonghua 11 background is able to confer strong resistance to African Xoo strain AXO1947 and Asian strain PXO86 with enhanced plant height and no yield penalty. These results imply that CR-S14 might be a better tester line than sweet 14 single-knockout mutant in the Kitaake background for diagnostic kit for rice blight resistance. The different disease responses between CR-S14 and the ossweet14 single knockout mutant in the Kitaake background might be due to the genetic background or off-target effect. This hypothesis needs to be experimentally confirmed. Moreover, natural nonfunctional OsSWEET14 mutant alleles need to be identified for further rice breeding applications. Finally, the enhancement of plant height needs to be taken into consideration when utilizing OsSWEET14 for resistant rice breeding.

\section{Methods}

\section{Rice growth conditions}

Zhonghua 11 (Oryza sativa L. ssp. Japonica cv. Zhonghua 11) was used in this study. CR-S14 is a genetically modified rice plants in the Zhonghua 11 background. At least 15 plants of different $C R$-S14 lines and more than 30 Zhonghua 11 plants were grown in a field under optimum growing conditions. We grew both CR-S14 and Zhonghua 11 for two seasons in 2019; the first season was from March to June, and the second season was from August to November. The paddy field is located in Guangzhou, Guangdong, China.

\section{Bacteria strains and inoculation}

All Xoo strains were cultivated on PSA medium $(10 \mathrm{~g} / \mathrm{l}$ peptone, $10 \mathrm{~g} / \mathrm{l}$ sucrose, $1 \mathrm{~g} / \mathrm{l}$ glutamic acid, $16 \mathrm{~g} / \mathrm{l}$ agar, and $\mathrm{pH}$ 7.0) at $28^{\circ} \mathrm{C}$ for 2 days. Bacteria were suspended in sterile water to an $\mathrm{OD}_{600} 0.5$ for inoculation on rice leaves. Bacterial blight inoculation was executed with the leaf-clipping method as previously described [29]. Inoculated rice leaves were collected $48 \mathrm{~h}$ after inoculation for gene induction analysis. Bacterial lesions were measured 14 days after inoculation. The sources of the Xoo strains tested in this study are listed in Additional file 7.

\section{Gene expression analysis}

The total RNA of rice leaves was extracted using an Eastep ${ }^{\circ}$ Super Total RNA Extraction Kit (Promega) and reverse-transcribed into single-stranded cDNA using GoScript $^{\text {tm }}$ Reverse Transcription Mix, Oligo (dT) (Promega, USA). Real-time quantitative RT-PCR was executed on LightCycler480 (Roche, Switzerland) using iTaq Universal SYBR Green Supermix (Bio-rad, USA). The expression of rice ubiquitin gene 5 (Ubi5) was used as the internal reference gene. The specific primer pair for Ubi5 was $5^{\prime}$-AACCACTTCGACCGCCACT-3' and 5 '-GTTCGATTTCCTCCTCCTTCC-3'. All the primers used in this study are listed in Additional file 8.

\section{Construct and rice transformation}

Two target sites were selected on the 1st and 3rd exon of OsSWEET14 and were inserted into the CRISPR/Cas9 vector as described [30]. A 2-kb promoter fragment upstream of the OSSWEET14 transcriptional initiation site was amplified and inserted in front of the GUS reporter gene in the pCAMBIA1301 vector. The plasmid was transformed into Agrobacterium EHA105. Rice transformation of Zhonghua 11 was performed as previously described [31].

\section{Determination of mutations in CR-S14}

The regions flanking the two targets on OSSWEE14 in independent $\mathrm{T}_{0}$ generation hygromycin-resistant CR-S14 lines were amplified with two pairs of primers, TargetTalC-F \& $R$ and Target-S14E3-F \& $R$, and were sequenced to determine the potential alterations. For those rice lines with biallelic mutations, modifications were determined in segregating progeny $\left(\mathrm{T}_{1}\right)$ of self-pollinated $\mathrm{T}_{0}$ plants. All the primers used in this study are listed in Additional file 8.

\section{Determination of $C R$-S14 morphology}

To determine the morphology of CR-S14, at least 15 plants of each line were measured. The upper diameter 
of the second stem segment of every productive tiller was measured as the stem diameter. The length of the mature rice plant from the ground to the tip of the panicle was measured as the plant height. The grain weight, number of filled grains and number of empty grains were weighed and counted with a seed morphology inspection machine (SC-G, wseen, China). Subsequently, the 1000-grain weight, seed setting rate and yield of the main panicle were calculated.

\section{Histochemical analysis of GUS activity}

Rice tissues were cut and transferred into a $2 \mathrm{ml}$ microcentrifuge tube containing GUS staining solution $(50 \mathrm{mM}$ sodium phosphate, $\mathrm{pH} 7.0,7 \%$ methanol, and $1 \mathrm{mM} 5$-bromo4-chloro-3-indolyl- $\beta$-d-glucuronide) overnight at $37^{\circ} \mathrm{C}$ and subsequently destained in an ethanol series. Stained rice tissues were observed under a stereomicroscope.

\section{Supplementary information}

Supplementary information accompanies this paper at https://doi.org/10. 1186/s12870-020-02524-y.

Additional file 1. Detection of mutations in CR-S14 transcripts.

Additional file 2. Prediction of transmembrane helices in OsSWEET14 and modified OSSWEET14.

Additional file 3. Predicted amino acid sequence of CR-S14.

Additional file 4. OsSWEET13 $\mathrm{ZH} 11_{1}$ EBE sequence.

Additional file 5. CR-S14 confers strong resistance to PXO86.

Additional file 6. Agronomic traits of CR-S14.

Additional file 7. Source of $X_{O O}$ strains.

Additional file 8. Primers used in this study.

\section{Acknowledgments}

We thank Dr. Yin Zhongchao from Temasek Life Sciences Laboratory for kindly providing the Xoo stains and Prof. Yaoguang Liu from South China Agricultural University for kindly providing the CRISPR/Cas9 vector system.

\section{Authors' contributions}

$X Z, Y L, N V, S S$ and $K X$ performed the experiments. $X Z$ and $M Z$ designed the project. XZ drafted the manuscript. All authors read and approved the final manuscript.

\section{Funding}

This work was supported by grants from the National Natural Science Foundation of China (31701403/31772384), National Key Research and Development Program of China (2017YFD0100101), the Chinese Academy of Sciences (XDA24030201), the Natural Science Foundation of Guangdong Province (2019A1515011975), and the Guangdong 'Pearl River Talents Plan'Postdoctoral Project. The funding bodies had no role in the design,

collection, and analysis, interpretation of data or in writing the manuscript.

\section{Availability of data and materials}

The datasets supporting the conclusions of this article are included within the article and its additional files.

\section{Ethics approval and consent to participate}

Not applicable.

\section{Consent for publication}

Not applicable.

\section{Competing interests}

The authors declare that they have no competing interests.

\section{Author details}

${ }^{1}$ Key Laboratory of South China Agricultural Plant Molecular Analysis and Genetic Improvement, Guangdong Provincial Key Laboratory of Applied Botany, South China Botanical Garden, Chinese Academy of Sciences, Guangzhou 510650, China. 'University of Chinese Academy of Sciences, Beijing 100049, China. ${ }^{3}$ Center of Economic Botany, Core Botanical Gardens, Chinese Academy of Sciences, Guangzhou 510650, China.

Received: 16 January 2020 Accepted: 25 June 2020

Published online: 03 July 2020

\section{References}

1. Duku C, Sparks AH, Zwart SJ. Spatial modelling of rice yield losses in Tanzania due to bacterial leaf blight and leaf blast in a changing climate. Clim Chang. 2016:135(3):569-83..

2. Niño-Liu DO, Ronald PC, Bogdanove AJ. Xanthomonas oryzae pathovars: model pathogens of a model crop. Mol Plant Pathol. 2006;7(5):303-24.

3. White FF, Potnis N, Jones JB, Koebnik R. The type III effectors of Xanthomonas. Mol Plant Pathol. 2009:10(6):749-66.

4. Scholze $H$, Boch J. TAL effectors are remote controls for gene activation. Curr Opin Microbiol. 2011;14(1):47-53.

5. Boch J, Scholze H, Schornack S, Landgraf A, Hahn S, Kay S, et al. Breaking the code of DNA binding specificity of TAL-type III effectors. Science. 2009; 326(5959):1509-12.

6. Moscou MJ, Bogdanove AJ. A simple cipher governs DNA recognition by TAL effectors. Science. 2009:326(5959):1501.

7. Streubel J, Pesce C, Hutin M, Koebnik R, Boch J, Szurek B. Five phylogenetically close rice SWEET genes confer TAL effector-mediated susceptibility to Xanthomonas oryzae pv. oryzae. New Phytol. 2013;200(3): 808-19.

8. Yang B, Sugio A, White FF. Os8N3 is a host disease-susceptibility gene for bacterial blight of rice. Proc Natl Acad Sci. 2006;103(27):10503-8.

9. Antony G, Zhou J, Huang S, Li T, Liu B, White FF, et al. Rice xa13 recessive resistance to bacterial blight is defeated by induction of the disease susceptibility gene Os11N3. Plant Cell. 2010;22(11):3864-76.

10. Zhou J, Peng Z, Long J, Sosso D, Liu B, Eom JS, et al. Gene targeting by the TAL effector PthXo2 reveals cryptic resistance gene for bacterial blight of rice. Plant J. 2015;82(4):632-43.

11. Yu Y, Streubel J, Balzergue S, Champion A, Boch J, Koebnik R, et al. Colonization of rice leaf blades by an African strain of Xanthomonas oryzae pv. oryzae depends on a new TAL effector that induces the rice nodulin-3 Os11N3 gene. Mol Plant-Microbe Interact. 2011;24(9):1102-13.

12. $X u$ Z, Xu X, Gong Q, Li Z, Li Y, Wang S, et al. Engineering broad-spectrum bacterial blight resistance by simultaneously disrupting variable TALEbinding elements of multiple susceptibility genes in rice. Mol Plant. 2019; 12(11):1434-46.

13. Oliva R, Ji C, Atienza-Grande G, Huquet-Tapia JC, Perez-Quintero A, Li T, et al. Broad-spectrum resistance to bacterial blight in rice using genome editing. Nat Biotechnol. 2019:37(11):1344-50.

14. Pavan $\mathrm{S}$, Jacobsen E, Visser RG, Bai Y. Loss of susceptibility as a novel breeding strategy for durable and broad-spectrum resistance. Mol Breed. 2010;25(1):1

15. Li T, Liu B, Spalding MH, Weeks DP, Yang B. High-efficiency TALENbased gene editing produces disease-resistant rice. Nat Biotechnol. 2012;30(5):390-2

16. Hutin M, Sabot F, Ghesquière A, Koebnik R, Szurek B. A knowledge-based molecular screen uncovers a broad-spectrum OSSWEET14 resistance allele to bacterial blight from wild rice. Plant J. 2015;84(4):694-703.

17. Blanvillain-Baufumé S, Reschke M, Solé M, Auguy F, Doucoure H, Szurek B, et al. Targeted promoter editing for rice resistance to Xanthomonas oryzae pv. oryzae reveals differential activities for SWEET 14-inducing TAL effectors. Plant Biotechnol J. 2017:15(3):306-17.

18. Eom JS, Luo D, Atienza-Grande G, Yang J, Ji C, Thi Luu V, et al. Diagnostic kit for rice blight resistance. Nat Biotechnol. 2019:37(11):1372-9.

19. Chen LQ, Hou BH, Lalonde S, Takanaga H, Hartung ML, Qu XQ, et al. Sugar transporters for intercellular exchange and nutrition of pathogens. Nature. 2010;468(7323):527-32 
20. Chen $L Q, Q u X Q$, Hou BH, Sosso D, Osorio S, Fernie AR, et al. Sucrose efflux mediated by SWEET proteins as a key step for phloem transport. Science. 2012;335(6065):207-11.

21. Bezrutczyk M, Yang J, Eom J-S, Prior M, Sosso D, Hartwig T, et al. Sugar flux and signaling in plant-microbe interactions. Plant J. 2018;93(4):675-85.

22. Braun DM. SWEET! The pathway is complete. Science. 2012;335(6065):173-4.

23. Chen LQ. SWEET sugar transporters for phloem transport and pathogen nutrition. New Phytol. 2014;201(4):1150-5.

24. Eom JS, Chen LQ, Sosso D, Julius BT, Lin IW, Qu XQ, et al. SWEETs, transporters for intracellular and intercellular sugar translocation. Curr Opin Plant Biol. 2015;25:53-62

25. Krogh A, Larsson B, Heijne G, Sonnhammer ELL. Predicting transmembrane protein topology with a hidden markov model: application to complete genomes. J Mol Biol. 2001;305(3):567-80.

26. Sato $Y$, Takehisa $H$, Kamatsuki K, Minami H, Namiki N, Ikawa $H$, et al. RiceXPro version 3.0: expanding the informatics resource for rice transcriptome. Nucleic Acids Res. 2012:41(D1):D1206-D13.

27. Tran TT, Pérez-Quintero AL, Wonni I, Carpenter SCD, Yu Y, Wang L, et al. Functional analysis of African Xanthomonas oryzae pv. oryzae TALomes reveals a new susceptibility gene in bacterial leaf blight of rice. PLOS Pathogens. 2018;14(6):e1007092.

28. Wang W, Mauleon R, Hu Z, Chebotarov D, Tai S, Wu Z, et al. Genomic variation in 3,010 diverse accessions of Asian cultivated rice. Nature. 2018; 557(7703):43-9.

29. Kauffman HE, Reddy APK, Hsieh SPY, Merca SD. An improved technique for evaluating resistance of rice varieties to Xanthomonas oryzae. Plant Dis Rep. 1973;57(6):537-41.

30. Ma X, Zhang Q, Zhu Q, Liu W, Chen Y, Qiu R, et al. A robust CRISPR/Cas9 system for convenient, high-efficiency multiplex genome editing in monocot and dicot plants. Mol Plant. 2015;8(8):1274-84.

31. Hiei Y, Ohta S, Komari T, Kumashiro T. Efficient transformation of rice (Oryza sativa L.) mediated by Agrobacterium and sequence analysis of the boundaries of the T-DNA. Plant J. 1994:6(2):271-82.

\section{Publisher's Note}

Springer Nature remains neutral with regard to jurisdictional claims in published maps and institutional affiliations.

Ready to submit your research? Choose BMC and benefit from:

- fast, convenient online submission

- thorough peer review by experienced researchers in your field

- rapid publication on acceptance

- support for research data, including large and complex data types

- gold Open Access which fosters wider collaboration and increased citations

- maximum visibility for your research: over $100 \mathrm{M}$ website views per year

At $\mathrm{BMC}$, research is always in progress.

Learn more biomedcentral.com/submissions 